\title{
Addressing Challenges in the Management of Paediatric Intussusceptions in the District Hospital
}

\author{
Senyo Gudugbe', Jonathan Cuthbert Balea Dakubo², Samuel Essoun ${ }^{3}$ \\ ${ }^{1}$ Holy Family Hospital, Techiman, Ghana \\ ${ }^{2}$ Department of Surgery, School of Medicine and Dentistry, University of Ghana, Accra, Ghana \\ ${ }^{3}$ Department of Surgery, Korle Bu Teaching Hospital, Accra, Ghana \\ Email: senyomd@gmail.com, jdakubo@yahoo.com, essoun@gmail.com
}

Received 20 August 2014; revised 18 September 2014; accepted 14 October 2014

Copyright (C) 2014 by authors and Scientific Research Publishing Inc.

This work is licensed under the Creative Commons Attribution International License (CC BY). http://creativecommons.org/licenses/by/4.0/

(c) (i) Open Access

\begin{abstract}
Intussusception is the leading cause of intestinal obstruction in children and its management can be challenging especially in centres with inadequate resources where this problem becomes more daunting. Nineteen cases of intussusception in the paediatric age group which were managed by medical officers in a district Hospital in Ghana are discussed. The outcomes of cases treated by open surgery, and those managed by pneumatic reduction were studied. We highlight the use of an improvised set up used in achieving pneumatic reduction thereby obviating operative reduction.
\end{abstract}

\section{Keywords}

Intestinal Obstruction, Pneumatic Reduction, Developing Country, Paediatric Surgery

\section{Introduction}

Intussusception, the telescoping of a proximal bowel segment into the adjoining intestinal lumen, is the leading cause of intestinal obstruction in children below 2 years [1] [2]. Its management can be challenging especially in a district hospital. In children it presents with a classic triad of vomiting, colicky abdominal pain, and bloody mucoid diarrhoea. Two-thirds of children with intussusception are younger than one year with peak incidence in infants aged 5 - 10 months [3]. The male:female incidence ratio is 3:1 [4]. The prognosis in patients with intussusception is excellent if diagnosed and treated early otherwise severe complications and death do occur. When diagnosed early, with appropriate fluid resuscitation and therapy, the mortality rate associated with intussusception in children is less than $1 \%$ but it is uniformly fatal if left unattended for more than 2 days [2].

How to cite this paper: Gudugbe, S., Dakubo, J.C.B. and Essoun, S. (2014) Addressing Challenges in the Management of Paediatric Intussusceptions in the District Hospital. Open Journal of Pediatrics, 4, 257-261. 
Surgery has been the main therapy in paediatric intussusception in most hospitals in deprived communities in Ghana and Africa with high resection rates due to late presentation [5]-[7]. Non operative management is hardly practiced because this requires fluoroscopy and ultrasonography which are largely unavailable here. Non-operative reduction of paediatric intussusception is done in Ghana on selected patients in the two main tertiary institutions in the country. Abantanga et al. in 2008 concluded that pneumatic reduction of intussusception in children without peritonitis is possible, practical, and reliable and must be tried first, preferably under general anaesthesia in the West African sub-region before proceeding to laparotomy in case of failure [8]. Done under fluoroscopic guidance it is cost-effective and a rapid method of managing intussusceptions [9].

Patients in rural and district communities usually present with late diseases and typically will not accept referrals to the tertiary centers. Confronted with this challenge the resident doctor has to do all that is possible to save lives. Paediatric intussusception has therefore been treated operatively, as is done in adult cases, in patients fit for surgery in this hospital. Four cases treated non-operatively without fluoroscopic support are presented here as an alternative method of treating these children to serve as a model that could be learnt and practiced in other district Hospitals in the developing world.

\section{Materials and Methods}

The study is a retrospective review of all consecutive cases of paediatric intussusceptions below age three managed at the district hospital between Jan 2008-Dec 2013, in Techiman in the Brong Ahafo Region of Ghana. The data was obtained from the theatre log and the patients' charts. The protocol for this research was approved by the constituted ethics committee of the Holy Family Hospital in Techiman, Brong Ahafo, Ghana where the study was carried out. The patients were categorized into those who had operative intervention versus nonoperative reduction. Clinical diagnosis was confirmed by an ultrasound scan showing either a target sign or a pseudokidney. Abdominal radiographs were done to exclude bowel perforation. Informed consent was obtained from parents.

All the patients were resuscitated with intravenous fluids and electrolyte. They were also given intravenous antibiotics and intra-nasal oxygen. Interventions to reduce intussusception were instituted immediately the patients became fit and stable enough for the procedure. The patients for non-operative reduction were strictly selected based on the following criteria: patient less than 3 years and onset of symptoms less than 48 hrs, absence of fever and/or peritonitis, normal white cell count. The outcome measures were complete reduction, morbidity and mortality.

The device for the non-operative reduction of the intussusception comprised the following: a hand bulb and aneroid gauge of a sphygmomanometer, a Foley's urethral catheter (24 F), a clamp and nasogastric tube connected to a saline filled kidney dish placed at a level below the patients' trunk.

The Foley's urethral catheter is lubricated and inserted into the rectum and the balloon inflated with 30 - 40 $\mathrm{ml}$ of air. It is then connected to both the hand bulb and the aneroid gauge of the sphygmomanometer which reads the pressure in the rectum (Figure 1). Air is insufflated into the rectum to a maximum of $120 \mathrm{mmHg}$ as defined by previous studies as the safe pressure to use [8] [10]. The patients gluteal folds were strapped together to prevent air leakage. The appearance of air bubbles in the saline filled kidney dish indicates successful reduction. A maximum of three attempts were allowed.

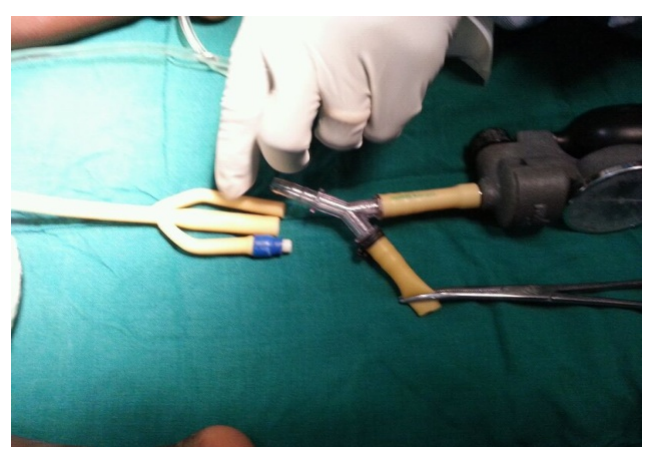

Figure 1. The setup of the pneumatic device showing the aneroid gauge of a sphygmomanometer, a connecting "Y” plastic tube and a $24 \mathrm{~F}$ three way urethral catheter and an artery forceps sealing off the "Y" tube. 
The device works on the basic principle that in the absence of an additional mechanical bowel obstruction from another cause, air insufflated per rectum should be able to travel through the hollow tube (bowel) and exit through a nasogastric tube; the ileocaecal valve having been compromised by the intussusceptum. A competent ileo-caecal valve may cause failure to demonstrate the air bubble in a completely reduced colo-colic intussuscetion and thus be a source of confusion. The amount of air insufflated into the rectum is gauged using the sphygmomanometer.

\section{Results}

Paediatric surgical cases represented 3\% (291/9462) of all surgical cases in the hospital during the study period. A total of 19 cases with 20 procedures were treated over the six year period, 15 had operative intervention, 4 had pneumatic reduction. These represented 6\% (19/291) of all paediatric surgical cases and were the only documented incidents of paediatric intestinal obstruction over the period. $63.2 \%$ (12) were males and $36.8 \%$ (7) were females with a male-to-female ratio of 1.7:1 (Table 1). The duration of symptoms ranged from 1 - 6 days with an average of 3.5 days and a mode of 3 days. $63.2 \%$ (12) of the cases presented after 3 days of symptoms (Table 2). $78.9 \%$ (15/19) of patients had operative intervention. $21.1 \%(4 / 19)$ had non operative reduction with one failure. The patient with unsuccessful pneumatic reduction had surgery but died later. For patients who had operative reduction, 56.3\% (9) had an ileo-colic intussusceptions and 43.7\% (7) were ileocaecal. Right hemicolectomy was done in 50\% (8) of operated cases (Table 3). The overall length of stay till discharge or death ranged from 2 - 14 days with an average of 5.12 days. Average lengths of stay for the non-operative and operative cases were 3.6 days and 4.7 days respectively (Table 4). 63.2\% (12) patients died and 36.8\% (7) survived. Only $26.6 \%$ (4) of the 15 patients who had surgical intervention survived while all the three cases who had successful non-operative reduction survived.

Table 1. Age and sex distribution.

\begin{tabular}{ccccccccccc}
\hline & \multicolumn{7}{l}{ Age in Months } \\
\hline Sex & 3 & 4 & 5 & 6 & 7 & 8 & 9 & 12 & Total \\
Female & 1 & 0 & 2 & 1 & 1 & 0 & 0 & 2 & 7 \\
Male & 0 & 3 & 2 & 3 & 1 & 1 & 1 & 1 & 12 \\
TOTAL & 1 & 3 & 4 & 4 & 2 & 1 & 1 & 3 & 19 \\
\hline
\end{tabular}

Table 2. Number of days before presentation.

\begin{tabular}{cccc}
\hline & Sex & & Total \\
\hline Days before Intervention & Female & Male & 3 \\
\hline 1 & 0 & 3 & 4 \\
2 & 2 & 2 & 5 \\
3 & 2 & 3 & 2 \\
4 & 0 & 1 & 4 \\
5 & 3 & 1 & 1 \\
6 & 0 & 12 & 19 \\
\hline
\end{tabular}

Table 3. Intra-operative findings.

\begin{tabular}{ccccc}
\hline & Outcomes & Total \\
\hline Intraop Findings & Alive & Died & 7 & 9 \\
\hline Ileo-Caecal & 2 & 5 & 8 & 16 \\
Ileo-Colic & 1 & 12 & \\
TOTAL & 4 & &
\end{tabular}


Table 4. Length of stay after surgery.

\begin{tabular}{cccc}
\hline & Sex & & Total \\
\hline Days post surgery & Female & Male & 3 \\
\hline 2 & 2 & 1 & 6 \\
3 & 0 & 6 & 2 \\
5 & 1 & 1 & 5 \\
8 & 1 & 4 & 1 \\
10 & 1 & 0 & 1 \\
12 & 1 & 0 & 1 \\
TOTAL & 1 & 0 & 19 \\
\hline
\end{tabular}

\section{Discussion}

The management of paediatric intussusceptions in the district hospital can be challenging. Treatment modalities include operative and non operative reduction. Non operative pneumatic reduction is associated with fewer complications and high success rates [10]. In Ghana, nonsurgical reduction was not started until January 2004 when it was commenced at Komfo Anokye Teaching Hospital (KATH) [8]. The difference between the procedures as done in KATH and Holy Family Hospital in Techiman is the former monitored their reductions under continuous ultrasound guidance whilst the latter had to rely on the appearance of bubbles through the nasogastric tube [8]. Further KATH had qualified paediatric surgeons, radiologists and anaesthesiologist while the Holy Family Hospital had only general Medical officers and nurse anaesthetists. The ages of patients ranged $3-12$ months with male preponderance; ratio 1.7:1. The average duration of symptoms before presentation was 3.5 days. Patients sought medical help from traditional healers, chemical sellers or other peripheral health centers where a diagnosis could not be made before reporting to the district hospital. This late presentation excludes patients from non operative intervention because of established sepsis. Of the 15 patients who had surgical intervention, 12 presented on or after three days and eventually had bowel resection and anastomosis with high mortality. Four patients who did not have peritonitis underwent pneumatic reduction with one failure. This implies that the longer the duration of symptoms, the higher the chance of established peritonitis with an attendant increased risk of bowel resection. The overall bowel resection rate was $42.1 \%$ which is within the $36 \%$ - $66 \%$ reported in other studies [5]-[7].

The overall mean length of stay was 5 days (range 2 - 14 days); 7 and 4.7 days respectively for the non-operative and operated cases. Length of stay was longer in the non-operative group. The average length of hospital stay in KATH was 2.3 days which compares with other published data in the literature of 2 days [11] [12]. The patients in the non operative group recovered their bowel function slower after the reduction, and for fear of discharging a patient with an incompletely reduced bowel or one that is perforated into the community these patients were kept a little longer for observation.

Only 4 (26.6\%) of the 15 patients who had surgical intervention survived. Overall 7 (36.8\%) of the 19 patients survived giving an unacceptably high mortality rate of $63.2 \%$.

3 (75\%) of the four pneumatic reductions were successful. All three patients who had successful pneumatic reduction survived. This success rate is comparable to those recorded KATH and the Korle Bu Teaching Hospital in Ghana [8] [9].

The device is cheap, easily assembled using materials already available in a hospital setting, can safely be employed without a fluoroscopy in the district hospital setting and the procedure can be carried out comfortably by a medical officer.

Limitations of this study include the small number of patients and selection bias for patients considered for non operative reduction. This can be addressed by increasing numbers and widening the scope of the research to other district hospitals.

\section{Conclusions}

Laparotomy for uncomplicated intussusception in children is attended by significant morbidity and mortality. 
The treatment of acute intussusception in many African countries remains predominantly surgical. The lack of qualified personnel, expertise and necessary equipment contributes to the limited use of pneumatic reduction in the district setting. When patients report early many cases can be successfully managed by pressure reduction and children should not be denied the benefits of this form of treatment [13]. Pneumatic reduction of intussusception is a choice that can be offered to patients in resource deprived setting using this improvised pneumatic reduction device.

Pneumatic reduction of intussusceptions in children is a viable and cost effective option for patients in the district setting in the absence of a fluoroscope.

This simple, effective, cheap and safe pneumatic device can be used to achieve reduction in selected patients thereby reducing surgical intervention.

Mortality from intussusceptions remains high in district hospital setting in Ghana with late presentation being the major contributing factor.

\section{Acknowledgements}

We will like to acknowledge the cooperation and contribution of Mr. Christopher Akanbobnaab, administrator and head of the Hospital Management team in the Holy Family Hospital towards this clinical work.

\section{References}

[1] Lloyd, D.A. and Kenny, S.E. (2004) The Surgical Abdomen. In: Walker, W.A., Goulet, O., Kleinman, R.E., et al., Eds, Pediatric Gastrointestinal Disease: Pathopsychology, Diagnosis, Management, 4th Edition, BC Decker, Ontario, 604.

[2] DiFiore, J.W. (1999) Intussusception. Seminars in Pediatric Surgery, 8, 214-220.

[3] Blanco, F.C., Cuffari, C., Chahine, A.A., King, L., Wilkes, G., Nazer, H., Windle, M.L., Li, B.U.K. and Young, M.G. Intussusception. http://emedicine.medscape.com/article/930708-overview

[4] Simpson, T., Ivey, J. and Borkowski, S. (2004) Pediatric Management Problems. Intussusception. Pediatric Nursing, 30, 326-327.

[5] Nmadu, P.T. (1992) The Changing Pattern of Intussusception in Northern Nigeria: An Analysis of 85 Consecutive Cases. East African Medical Journal, 69, 640-642.

[6] Munkonge, L. (1983) Experience in the Management of Intussusception in Zambian Children. Medical Journal of Zambia, 17, 56-58.

[7] Postma, M.H. and Hadley, G.P. (1985) Intussusception in Black Children. South African Medical Journal, 68, 405406.

[8] Abatanga, F.A., et al. (2008) Pneumatic Reduction of Intussusception in Children at the Komfo Anokye Hospital, Kumasi, Ghana. East African Medical Journal, 85, 550-555.

[9] Mensah, Y.B., et al. (2011) Pneumatic Reduction of Intussusception in Children at Korle Bu Teaching Hospital: An Initial Experience. African Journal of Paediatric Surgery, 8, 176-181.

[10] Zulfiqar, M.A., Noryati, M., Hamzaini, A.H. and Thambidorai, C.R. (2006) Pneumatic Reduction of Intussusception Using Equipment Readily Available in the Hospital. Medical Journal of Malaysia, 61.

[11] Gilmore, A.W., Reed, M. and Tenenbein, M. (2010) Management of Childhood Intussusception after Reduction by Enema. The American Journal of Emergency Medicine.

[12] Herwig, K., Brenkert, T. and Losek, J.D. (2009) Enema-Reduced Intussusception Management: Is Hospitalization Necessary? Pediatric Emergency Care, 25, 74-77. http://dx.doi.org/10.1097/PEC.0b013e318196ea2d

[13] Ameh, E.A. (2002) The Morbidity and Mortality of Laparotomy for Uncomplicated Intussusception in Children. West African Journal of Medicine, 21, 115-116. 
Scientific Research Publishing (SCIRP) is one of the largest Open Access journal publishers. It is currently publishing more than 200 open access, online, peer-reviewed journals covering a wide range of academic disciplines. SCIRP serves the worldwide academic communities and contributes to the progress and application of science with its publication.

Other selected journals from SCIRP are listed as below. Submit your manuscript to us via either submit@scirp.org or Online Submission Portal.
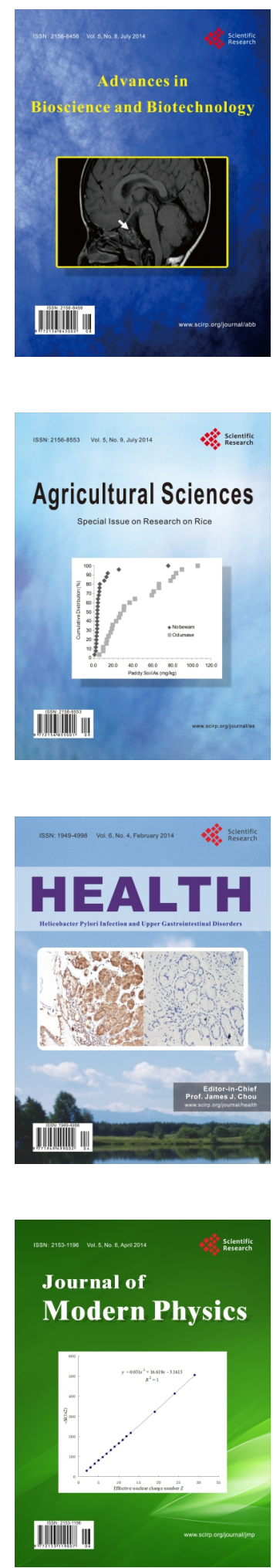
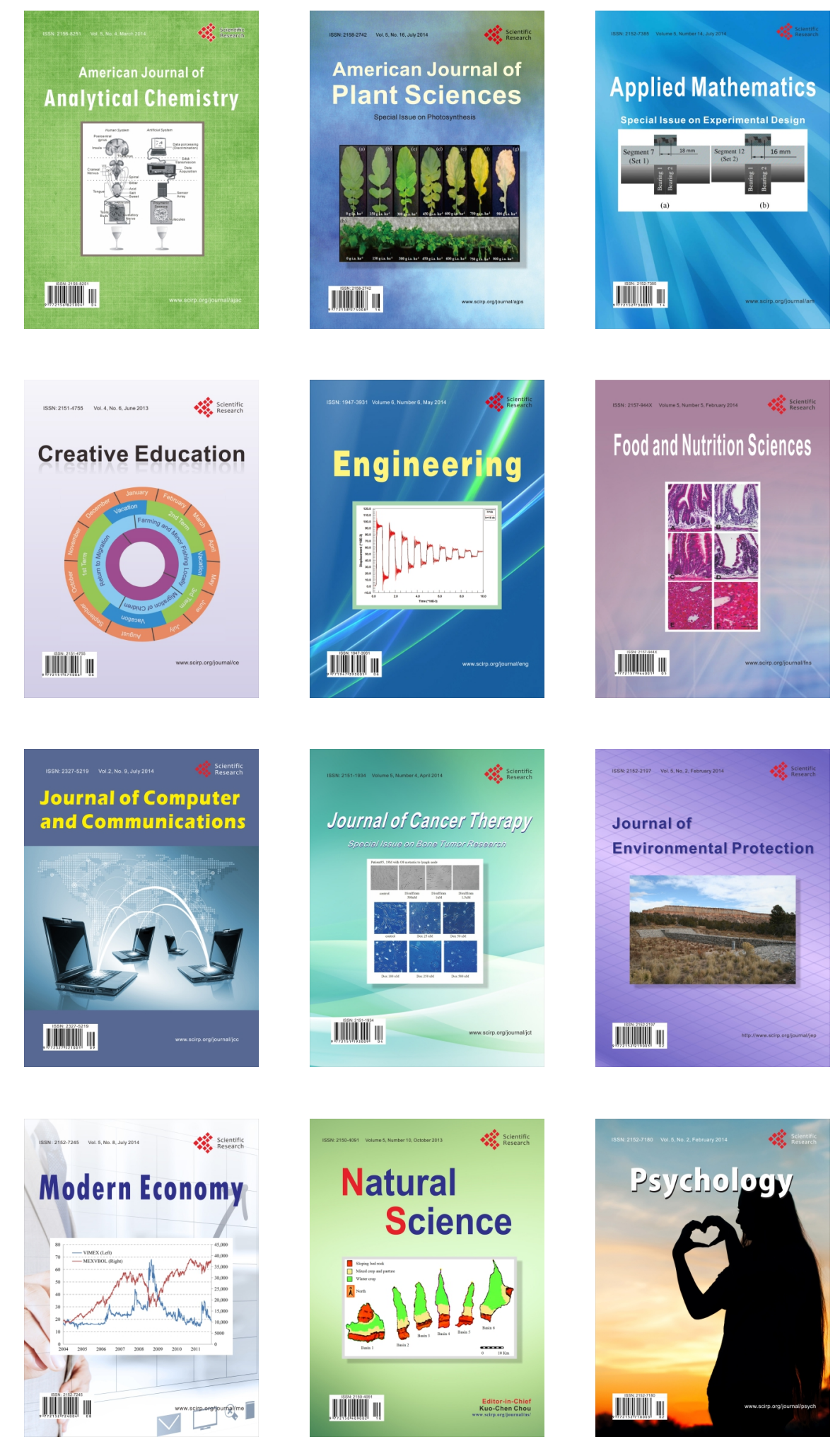